\title{
Derrame Pericárdico, Enfrentamiento Clínico
}

\author{
CRISTIÁN CLAVERÍA R. ${ }^{1}$, LUIS VERGARA G. ${ }^{2}$, SANDRA NEGRÓN M. ${ }^{3}$, \\ CARMEN LUZ LÓPEZ L. ${ }^{3}$, PAMELA ZELADA P. ${ }^{4}$, JUAN ANDRÉS CARRASCO O. ${ }^{5}$ \\ 1. Cardiólogo Pediatra, Facultad de Medicina Pontificia Universidad Católica de Chile y Hospital Padre Hurtado, SSMSO. \\ 2. Interno de Medicina, Facultad de Medicina Pontificia Universidad Católica de Chile. \\ 3. Becado de Cardiología, Facultad de Medicina Pontificia Universidad Católica de Chile. \\ 4. Cardiólogo Pediatra, Facultad de Medicina Pontificia Universidad Católica de Chile y Hospital Dr. Sótero del Río, SSMSO. \\ 5. Intensivista Pediátrico, Facultad de Medicina, Pontificia Universidad Católica de Chile.
}

\section{ABSTRACT \\ Clinical Approach to Pericardial Effusion}

Pericardial effusion is the main complication of pericarditis, and can create serious consequences depending on the speed of development and etiology. The most common causes are neoplasms and idiopathic, even though viral etiology is a frequently underestimated cause because of the difficulty in its confirmation. In cases of significant pericardial effusion, pericardiocenthesis has demonstrated to be an effective and safe procedure. We present a clinical case of a 14 year old adolescent who complained of persistent abdominal pain, and whose study showed significant pericardial effusion. Pericardiocenthesis was performed, along with installation of a pigtail catheter which drained $500 \mathrm{ml}$ of serohematic pericardial effusion. The etiologic study included an PCR (polymerase chain reaction) positive for Enterovirus. The patient recovered favorably and was dismissed from the hospital in good condition. The objective of this report is to expose a current revision of the clinical management of pericardial effusion and the technical skills of pericardiocenthesis. Knowledge of the technique is fundamental in the treatment of pericardial effusion, especially in situations associated with hemodynamic compromise.

(Key words: Pericarial effusion, pericardiocenthesis technique ).

Rev Chil Pediatr 2009; 80 (3): 267-273

\section{RESUMEN}

El derrame pericárdico es la principal complicación de la pericarditis, la cual puede generar graves consecuencias dependiendo de la velocidad de instauración como de su etiología. Las principales causas reportadas son las neoplásicas y las idiopáticas, sin embargo, la etiología viral es subestimada debido a la dificultad de confirmar el diagnóstico. En casos de derrame pericárdico significativo la pericardiocentesis ha mostrado ser un procedimiento eficaz y seguro. Presentamos caso clínico de un adolescente de 14 años quién consulta por dolor abdominal persistente, cuyo estudio demuestra un derrame pericárdico. Se realizó pericardiocentesis e instalación de catéter pigtail dando salida a $500 \mathrm{~mL}$ de líquido pericárdico serohemático. Dentro del estudio etiológico presenta PCR (Reacción de Polimerasa en cadena) positivo para enterovirus.

Trabajo recibido el 09 de octubre de 2008, devuelto para corregir el 12 de enero de 2009, segunda versión el 19 de abril de 2009, aceptado para publicación el 04 de mayo de 2009.

Correspondencia a:

Dr. Cristián Clavería R.

E-mail: claveria@med.puc.cl 
Paciente evoluciona favorablemente y es dado de alta en buenas condiciones. El objetivo del presente reporte es exponer la revisión actualizada del enfrentamiento clínico del derrame pericárdico y la técnica de pericardiocentesis. El conocimiento de la técnica de pericardiocentesis en fundamental en el tratamiento del derrame pericárdico, en especial en situaciones con compromiso hemodinámico.

(Palabras clave: Derrame pericárdico, técnica pericardiocentesis).

Rev Chil Pediatr 2009; 80 (3): 267-273

El pericardio es un doble saco membranoso, fibroso y seroso que envuelve al corazón y al origen de los grandes vasos. Esta compuesta por una capa interna o visceral serosa y una capa externa o parietal fibrosa ${ }^{1,2}$.

El espacio pericardio esta localizado entre la capa parietal y visceral. Normalmente contiene entre 5 a $20 \mathrm{ml}$ de fluido claro que actúa como lubricante permitiendo el movimiento libre del corazón dentro del saco pericárdico.

La inflamación (pericarditis) o lesión del espacio pericárdico puede ser agudo o crónico. Se observa precoz en la evolución de infarto miocárdico, cirugía cardíaca y trauma cerrado o penetrante. Otras causas incluyen reacción inmunológica autoinmune o en respuesta a infección viral previa.

Complicación más importante secundaria a una pericarditis es el Derrame Pericárdico (DP). Este es particularmente importante cuando existe acumulación de líquido en forma súbita produciendo taponamiento cardíaco. Dependiendo del tamaño del paciente, cantidades tan pequeñas de líquido como $20 \mathrm{ml}$, acumulado en minutos $u$ horas puede causar taponamiento, mientras que derrames subagudos y crónicos tan significativos como 1 a 2 litros pueden ser muy bien tolerados en niños mayores y adultos.

El DP puede ser secundario a infecciones, falla cardíaca congestiva, estado post cirugía de Fontan, Síndrome post pericardiotomía y algunas enfermedades neoplásicas.

El objetivo del presente reporte es exponer la revisión actualizada del enfrentamiento clínico del derrame pericárdico y la técnica de pericardiocentesis.

\section{Caso clínico}

Adolescente de 14 años, sexo masculino, eutrófico, con antecedentes de Síndrome Autista en tratamiento con risperidona y retraso del desarrollo psicomotor. Consultó por cuadro de 2 semanas de evolución caracterizado por dolor abdominal difuso asociado a diarrea y fiebre. El paciente fue evaluado ambulatoriamente y tratado con antiespasmódicos y omeprazol. El cuadro clínico persistió, con incremento del dolor abdominal, que se intensificaba al estar en decúbito supino. Consulta en Hospital Sótero del Río donde destacaba paciente inquieto, con preferencia por estar sentado y dolor a la palpación en hipocondrio derecho, ambos flancos y en fosa iliaca derecha. Se indicó radiografía de tórax (figura 1) en la que destacaba gran cardiomegalia, con Indice Cardiotoráxico de 0,71 (valor normal menor a 0,4). Ecocardiograma mostró gran derrame pericárdico, sin deformidad de aurículas en diástole y función sistólica del ventrículo izquierdo conservada. Se derivó al Hospital Clínico de la Universidad Católica para realización de pericardicentesis y estudio etiológico, ingresando en buenas condiciones

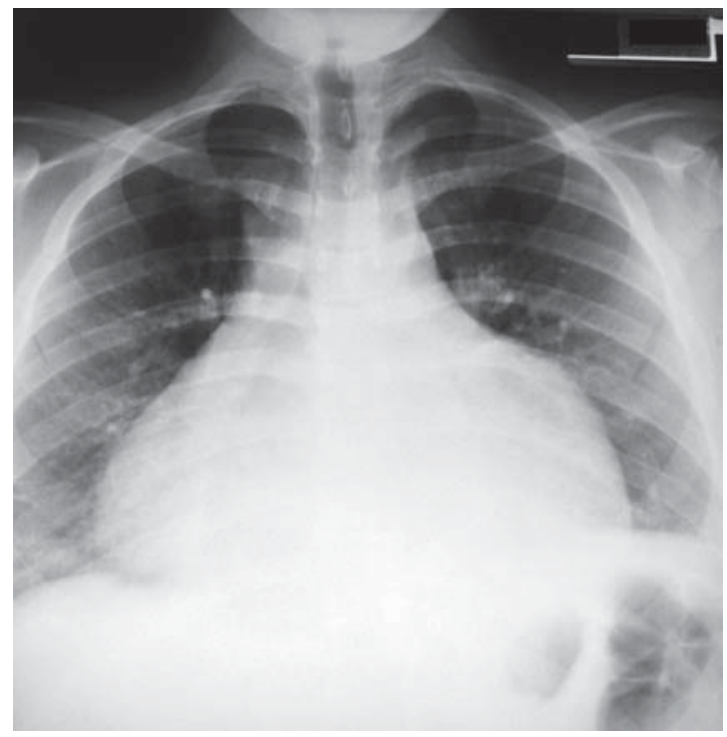

Figura 1. Radiografía de tórax Ap-Lat de ingreso Hospital Sótero del Río. 
generales, hemodinámicamente estable. Se realizó pericardiocentesis por punción percutánea que dio salida a abundante líquido serohemático, instalandose cateter pigtail de pericardiosentesis que da salida a un total de $500 \mathrm{~mL}$ de líquido pericárdico. Se inició tratamiento antibiótico con cloxacilina, cefotaximo y claritromicina. Exámenes generales normales. Estudio del líquido reveló cultivos, PCR (Reacción de Polimerasa en cadena) mycoplasma, PCR (Reacción de Polimerasa en cadena) micobacterias, C3, C4, Factor Reumatoideo, ANA y antiDNA negativos. Se solicitó PCR (Reacción de Polimerasa en cadena) para enterovirus que resultó positivo, suspendiéndose tratamiento antibiótico. Evolucionó favorablemente, con Ecocardiograma y radiografías seriadas de control que demostraron resolución del derrame pericárdico por lo cual se retiró catéter pigtail al séptimo día de evolución. Se indicó alta médica al décimo día de hospitalización, con control ambulatorio.

\section{Comentario}

\section{Derrame pericárdico}

El derrame pericárdico se define como la presencia de líquido en el espacio pericárdico superior a la normal (15-50 $\mathrm{ml} \mathrm{o} 1 \mathrm{ml} / \mathrm{kg})$ secundario a una pericarditis. Su origen puede deberse a una amplia variedad de situaciones clínicas tales como infecciones virales, bacterianas, fúngicas, o como resultado de procesos inflamatorios, autoinmunes, neoplásicos y dentro de la evolución postoperatorio de la cirugía cardíaca $^{3}$ (tabla 1). Hace 40 años atrás, la fiebre reumática y la pericarditis bacteriana fueron las etiologías más comunes ${ }^{4}$. Con la introducción de las terapias antibióticas y nuevas vacunas, en especial contra $H$. influenzae, las causas bacterianas han disminuido considerablemente. Actualmente, la frecuencia de las causas de derrame pericárdico depende de la población y lugar geográfico en que se haya estudiado ${ }^{1}$. Publicaciones recientes señalan que las causas más comunes serías las neoplásicas y las idiopáticas ${ }^{4}$. Sin embargo, la etiología viral todavía es una causa común en ciertos reportes ${ }^{5}$, la cual es subestimada debido a la dificultad de confirmar el diagnóstico. La técnica de reacción de polimerasa en cadena (PCR) es una herramienta diagnostica que permite identificar la etiología viral específica ${ }^{6}$. En pacientes VIH positivo es más común la pericarditis tuberculosa ${ }^{7}$.

\section{Clínica}

La presentación clínica está determinada por el aumento de la presión intrapericárdica, que depende de varios factores como la enfermedad de base, edad del paciente y velocidad de instalación del derrame ${ }^{2,3}$. La acumulación rápida de líquido es peor tolerado que la acumulación lenta, la cual se hace manifiesta al comprimir estructuras vecinas.

El principal síntoma de pericarditis aguda es el dolor precordial, el cual en pediatría se mani-

Tabla 1. Etiología de derrame pericárdico y tamponamiento

- Viral: Coxsackievirus B, echovirus, adenovirus, Epstein-Barr, parotiditis, varicela, poliomielitis, hepatitis B, influenza (incluso postvacuna), citomegalovirus, sarampión, virus respiratorio sincicial, VIH

- Bacteriana/Tuberculosis: Staphylococcus aureus, Haemophilus influenzae, Neisseria meningitidis, Streptococo pneumoniae (incluyendo formas resistentes), Streptococo B hemolítico, Micoplasma pneumoniae, Micobacterium tuberculosis.

- Micoticas: Cándida, aspergilus

Pericarditis con enfermedad sistémica: Enfermedad inflamatoria sistémica, fiebre reumática, artritis reumatoide juvenil, lupus eritematoso sistémico, colitis ulcerosa, enfermedad de Kawasaki. Neoplasmas: leucemia, linfoma, tumor metastásico, pericarditis por radiación. Renal: enfermedad renal terminal, diálisis.

Traumática y postoperatoria: Penetrante torácica, catéter central mal posicionado, cateterización y biopsia, ruptura de aneurisma coronario (Kawasaki), hemorragia temprana postoperatoria, síndrome postpericardiotomia, elevada presión venosa (post-Fontán), post transplante cardíaco

Drogas: Procainamida, hidralazina, penicilina, cromolin, dantrolene, anatraciclinas

Misceláneo: Presión venosa central elevada: Síndrome vena cava superior, hipertensión pulmonar primaria y falla cardíaca derecha, insuficiencia cardíaca congestiva descompensada
} 
fiesta de forma variable dependiendo de la edad del niño y su capacidad de manifestarlo. El dolor se irradia al cuello, ambos trapecios o epigastrio que se alivia al inclinarse hacia adelante y aumenta en decúbito supino, inspiración, deglutir o al movilizarse. En ocasiones hay dolor pleurítico por compromiso de la pleura adyacente. Generalmente, se presenta fiebre baja (mayor a $38^{\circ}$ en la pericarditis purulenta). Puede haber taquipnea y disnea al inicio del tamponamiento. En pericarditis aguda estos síntomas son precedidos por un pródromo viral o de infección respiratoria alta.

En el examen físico lo característico son los frotes pericárdicos, pero su ausencia no descarta la enfermedad. Además, el niño presenta intranquilidad, con movimiento precordial disminuido y tonos cardíacos apagados. En niños mayores puede haber ingurgitación yugular por elevación de la presión venosa central y hepatomegalia. Se puede encontrar pulso paradójico, que corresponde a la caída de la presión sistólica mayor de $10 \mathrm{mmHg}$ durante la inspiración. El compromiso hemodinámico es variable y dependerá de la velocidad de instalación y cuantía del derrame, pudiendo existir hipoperfusión con hipotensión, disminución o ausencia de pulsos periféricos con extremidades frías y oliguria en los casos más graves. En lactantes y niños pequeños, la taquicardia puede ser en ocasiones el único signo presente ${ }^{8}$. Se describe la triada de Beck caracterizada por: 1) caída de la presión arterial; 2) elevación de la presión venosa y 3 ) "corazón callado y quieto".

\section{Hallazgos de laboratorio}

En la radiografía de tórax se puede observar aumento de la silueta cardíaca, con imagen globular o apariencia de "botella de agua". En el ECG se puede observar alteraciones de la repolarización ventricular con desnivel ST e inversión de la onda T. La alternancia de la amplitud o bajo voltaje del QRS puede aparecer con derrame pericárdico severo.

El ecocardiograma constituye el principal instrumento diagnóstico, tanto para cuantificar la cuantía del derrame, presencia de tabicaciones, repercusión funcional o hemodinámica del derrame, con colapso de la aurícula y ventrículo derecho al final del diástole.
Tabla 2. Exámenes de laboratorio

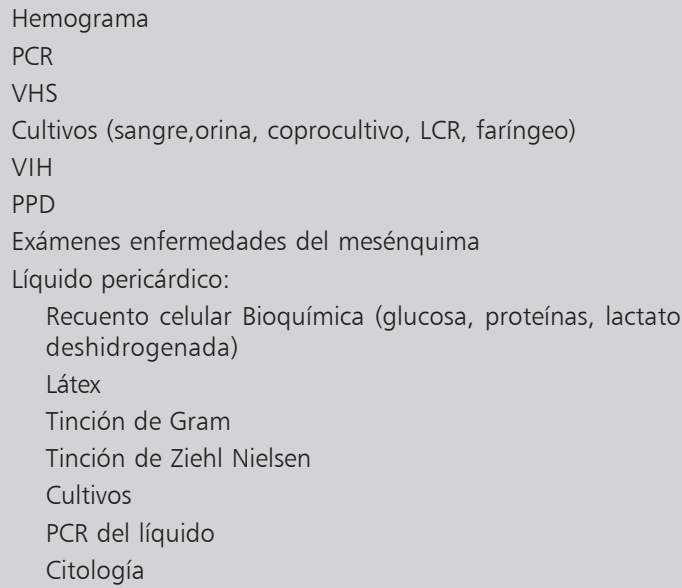

Los exámenes generales (tabla 2), como el hemograma puede mostrar leucocitosis de predominio polimorfonuclear en el caso de la pericarditis purulenta, y linfocitosis en el caso de la pericarditis viral o síndrome postpericardiotomía. La VHS y la PCR se encuentran habitualmente elevadas.

Las enzimas cardíacas pueden estar levemente elevadas debido a compromiso inflamatorio del miocardio.

Se deberían realizar los cultivos de sangre, orina, LCR, faringeo, nasofaringeo, coprocultivo en busca de patógenos virales o bacterianos. Considerar según antecedentes el test de VIH, PPD, exámenes para enfermedades del mesénquima. Estudio de líquido pericárdico que debería incluir: recuento celular, citología, bioquímica (glucosa, proteínas, lactato deshidrogenasa), látex, tinción de gram y de Ziehl Nielsen y cultivos. La PCR del líquido pericárdico puede ser útil en determinados casos. El examen citológico se debe realizar siempre para descartar etiología neoplásica. La biopsia pericárdica ha sido reportada en adultos pero en niños su rol es discutido.

Generalmente, el líquido pericárdico de la pericarditis purulenta muestra marcada leucocitosis o pus franca. En el líquido el nivel de proteínas es elevado y la concentración de glucosa es baja. En el líquido de pericarditis idiopática puede ser leve a completamente sanguinolento, con predominio de mononucleares. 


\section{Tratamiento}

El tratamiento general consta de un manejo de soporte, antiinflamatorios y el drenaje por pericardiocentesis, además del manejo específico dependiendo de la etiología. El pronóstico del derrame pericárdico principalmente depende de la etiología y del compromiso hemodinámico. En pacientes sin compromiso hemodinámico la pericardiocentesis sólo se justificaría en caso que entregue información relevante respecto a la etiología, pues es un procedimiento que tiene riesgo de complicaciones ${ }^{9}$. Además, en pacientes con derrame crónico, definido como la presencia de derrame moderado a severo al ecocardiograma luego de 3 meses después del diagnóstico o drenaje, fue similar entre el grupo que fue drenado por pericardiocentesis y quienes no lo hicieron (odds ratio $2,1,95 \%$ intervalo de confianza 0,7 a 2,1$)^{4}$.

\section{Soporte}

Debido a que el derrame afecta la precarga es importante aporte de volumen parenteral.

Los diuréticos pueden empeorar la condición del paciente al reducir la precarga.

El gasto cardíaco es mantenido por reflejo de taquicardia y aumento de la contractilidad. Los agentes inotrópicos pueden ser útiles. En casos más avanzados de tamponamiento, la presión sanguínea es mantenida por vasoconstricción periférica de los lechos arterial y venoso, por lo cual los vasodilatadores deberían ser evitados. Con tamponamiento más severos se puede producir paro cardiorrespiratorio y requerir intubación endotraqueal y ventilación mecánica. La aplicación de presión positiva, PEEP o CPAP deben realizarse con mucho cuidado debido a que el incremento de presión intratorácica y del aumento de volumen pulmonar puede reducir el retorno venoso sistémico y aumentar la constricción externa del corazón.

\section{Terapia específica}

El tratamiento de la pericarditis viral o idiopática consiste en reposo en cama y antiinflamatorios como aspirina o AINES. En casos refractarios pueden requerir tratamiento con prednisona. El síndrome postpericardiotomia tiene un manejo similar, prefiriéndose el uso de antiinflamatorios no esteroidales como el ibuprofeno por 4 a 6 semanas. La pericarditis idiopática usualmente remite espontáneamente o cede con antiinflamatorios; en caso que persista por más de 2 semanas se deberían investigar otras causas. La recurrencia tardía hace unos años se asoció a derrames idiopáticos y a causas inflamatorias autoinmunes. Sin embargo, estudios recientes no han identificado asociación entre recurrencia y alguna etiología específica ${ }^{4}$.

La pericarditis purulenta requiere tratamiento agresivo combinado con antibióticos, apoyo hemodinámico con drogas vasoactivas y drena$\mathrm{je}^{10}$. La aspiración con aguja permite mejoría hemodinámica inicial, pero el drenaje puede ser dificultoso con la aguja debido a la presencia de fibrina en el líquido. En pericarditis purulenta el tratamiento antibiótico inicial debería ser de amplio espectro incluyendo cobertura para Staphylococcus, H. influenzae, S. pneumoniae y $N$. meningitidis. La duración del tratamiento antibiótico es usualmente de 4 a 6 semanas. Se debe tener presente la posibilidad de pericarditis constrictiva aun después de un tratamiento exitoso.

El tratamiento de la pericarditis tuberculosa debería ser con combinación de tratamiento antituberculoso y en etapas precoces se recomienda asociar a prednisona para disminuir la inflamación y adhesión del pericardio. En pericarditis asociada con enfermedad sistémica, la pericardiocentesis y estudios citológicos como la evaluación sistemática de otros órganos son importantes para un adecuado diagnóstico. Sin apropiado manejo general el tratamiento estándar con antiinflamatorios y drenaje puede no ser efectivo.

\section{Técnica de pericardiocentesis}

El drenaje por pericardiocentesis fue descrita por primera vez en 1955 por Fallows and Pastor $^{11}$. Desde entonces numerosos reportes han demostrado la eficacia y seguridad de este procedimiento $^{12,13}$. La pericardiocentesis percútanea puede ser realizada de emergencia a ciegas o bien guíada por medio de ecocardiograma transtoráxico, el cual permite la instalación de un drenaje en forma más segura. Este procedimiento se debe realizar en la Unidad de 
Cuidados Intensivos o Laboratorio de Cateterismo Cardíaco, donde existan métodos de monitorización y medios adecuados de reacción frente a posibles complicaciones, lo que incluye manejo de vía aérea y apoyo cardiocirculatorio. El paciente debe contar con acceso venoso seguro en caso de requerir aporte de volumen y drogas de emergencia. Dosis de drogas de emergencia deben estar previamente definidas en hoja ad hoc de Reanimación Cardiopulmonar avanzada.

El ecocardiograma es útil para refinar el procedimiento y es parte esencial en la técnica de instalación ${ }^{8,14,15}$, pues permite determinar el sitio de punción, definir el curso de la aguja,

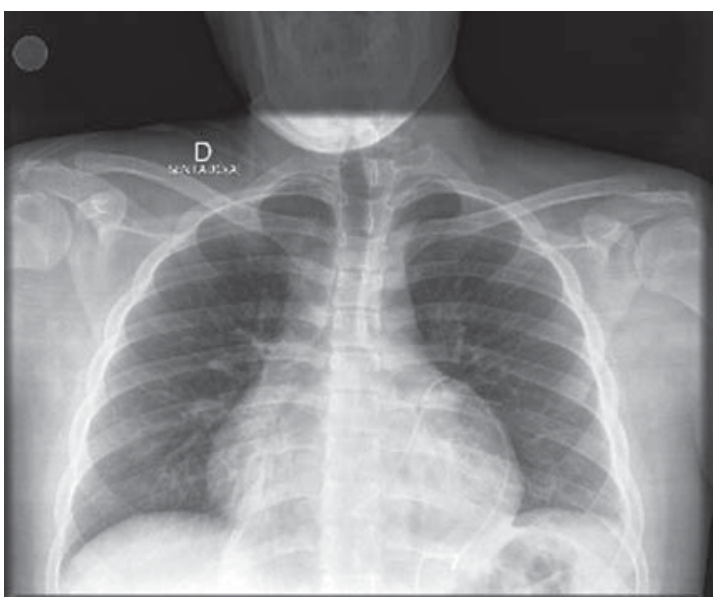

Figura 2. Radiografía de torax de control post pericardiocentesis. Flecha muestra cateter pigtail ubicado en espacio pericárdico.

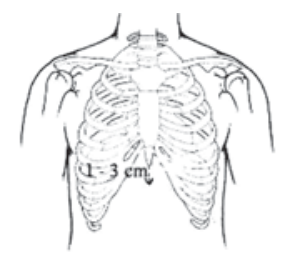

1: Identificar entre 1 a $3 \mathrm{~cm}$, dependiendo de la edad del paciente, distal a la apófisis xifoides en la linea media.

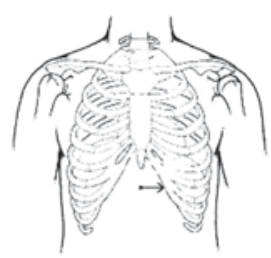

2: En este sitio, movilizarse hasta el reborde costal izquierdo. Éste es el sitio de punción.

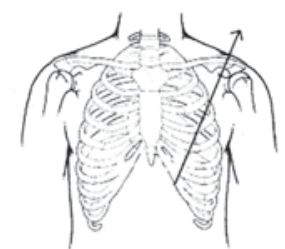

3: Insertar la aguja en dirección al hombro izquierdo, aspirando continuamente.
El ángulo de punción debe ser de $15^{\circ}$, previa infiltración con lidocaina. Una vez obtenido líquido se inserta $2 \mathrm{~cm}$ el teflón, retirando posteriormente la aguja.

Figura 3. Técnica de pericardiocentesis. Sitio de punción. determinar la profundidad y, mediante la inyección de solución fisiológica de constraste, se puede confirmar la localización intrapericardica de la aguja. El mejor sitio de punción ha sido descrito como el punto en el cual la mayor acumulación de líquidos es más cercana a la superficie corporal, siendo la localización subxifoidea izquierda la más frecuente (figuras 2 y $3)$. La aguja debe ser de un largo tal que alcance el derrame ( $6 \mathrm{~cm}$ puede ser adecuado), usando frecuentemente un Teflón números 16, 18 y/o 20 según características del paciente. Se utilizan: agujas, guías, dilatadores y catéteres.

El procedimiento se debe realizar bajo sedación, analgésia sistémica y anestesia local, con monitorización no invasiva continua la cual debe incluir: monitorización electrocardiográfica, presión arterial, saturación transcutánea de oxígeno.

Previo técnica de asepsia, la piel y estructuras más profundas son infiltradas con lidocaina $1-2 \%$. Se inserta la aguja con Teflón en el sitio previamente definido y siguiendo una trayectoria predeterminada con orientación hacia el hombro izquierdo, aspirando en forma contínua. El ángulo usual por acceso subxifoideo es de 15 grados sobre la piel. Si el líquido es obtenido, la vaina del Teflón se avanza cerca de $2 \mathrm{~cm}$ manteniendo fija la aguja y luego esta se remueve. Si el líquido obtenido es sanguinolento se debe confirmar la posición intrapericardica. Si esta es intrapericardico el líquido extraído no se coagula. Además se puede inyectar una pequeña cantidad de suero salino agitado como forma de obtener constraste para así verificar la posición por medio de la visión ecocardiográfica. La opacificación densa del espacio pericárdico confirma la posición intrapericardica (contraste salino) con ausencia de contraste intracardíaco.

El derrame puede ser drenado con punción aspirativa única. Si se requiere drenaje prolongado se debe introducir por el Teflón una guía con punta blanda para evitar daño sobre el corazón y asegurar la ubicación en el espacio pericárdico y así luego introducir catéter tipo 
pigtail, utilizando técnica Seldinger. Una vez que la guía es pasada ampliamente hacia el espacio pericárdico, el catéter teflón es removido. Luego se realiza una pequeña insición sobre la piel con bisturí para disminuir la resistencia que ejerce la piel y celular subcutáneo al paso del catéter pigtail. Se puede facilitar aun más el paso del catéter con la introducción previa de un dilatador. Estas maniobras facilitan el paso del catéter pigtail a través de los tejidos hacia el espacio pericárdico. Se procede posteriormente al drenaje del líquido mediante aspiraciones repetidas. El catéter pigtail se debe mantener conectado a sistema de aspiración suave continua, tipo Hemosuc, hasta que la producción de líquido pericárdico se encuentre en rango mínimo fisiológico.

Las principales complicaciones del procedimiento son el hemopericardio por punción intracardíaca si la aguja es dirigida posteriormente, la laceración del epicardio o del miocardio ventricular, laceración de una arteria o vena coronarias, la laceración de vasos mamarios, fibrilación ventricular, punción del esófago con mediastinitis subsecuente, el neumotórax, hematoma del sitio de punción y la infección de la cavidad pleural o pericárdica. La tasa de complicaciones reportado por Tsang fue de $1 \%$ de complicaciones mayores (neumotórax) y 3\% de complicaciones menores (punción ventrículo derecho y neumotórax pequeño que no requirieron tratamiento), lo cual se correlaciona con otros reportes ${ }^{16-18}$.

En resumen el conocimiento de la técnica de pericardiocentesis es fundamental para el adecuado enfrentamiento clínico del derrame pericárdico, sobre todo en situaciones que implican compromiso hemodinámico. Se demuestra que es una técnica eficaz y segura en su implementación.

\section{Referencias}

1.- Sagrista-Sauleda J, Merce J, Permanyer-Miralda G, Soler-Soler J: Clinical clues to the causes of large pericardial effusions. Am J Med 2000; 109 (95).

2.- Keane JF: NADAS' Pediatric Cardiology. 2a Edición ed. Vol. Capitulo 27, Pericardial Diseases. 2006.

3.- Nichols DG: Critical Heart Disease in Infants and Children. 2a edición ed. Vol. Cap. 9.'Pericardial
Effusion and Tamponade. 2006.

4.- Kuhn B, et al: Etiology, management, and outcome of pediatric pericardial effusions. Pediatr Cardiol 2008; 29 (1): 90-4.

5.- Maischa PMS B, Risticb AD, Erbelc R, et al: Guía de Práctica Clínica para el diagnóstico y tratamiento de las enfermedades del pericardio. Rev Esp Cardiol 2004; 57: 1090-114.

6.- Levy P, Fournier PE, Charrel R, et al: Molecular analysis of pericardial fluid: a 7-year experience. Eur Heart J 2006; 27.

7.- Mayosi B, Wiysonge CS, Ntsekhe M, et al: Clinical characteristics and initial management of patients with tuberculous pericarditis in the HIV era: the Investigation of the Management of Pericarditis in Africa (IMPI Africa) registry. BMC Infect Dis 2006; 6: 2.

8.- Tsang $T$, Freeman WK, Sinak LJ, Seward JB: Echocardiographically guided pericardiocentesis: Evolution and state-of-the-art technique. Mayo Clin Proc 1998; 73: 647.

9.- Soler-Soler J J: Sagrista-Sauleda, and G. PermanyerMiralda, Management of pericardial effusion. Heart 2001; 86 (2): 235-40.

10.- Moss A: Heart Disease in Infants, Children, and Adolescents. sixth ed. 2001.

11.- Fallows J, Pastor B: The use of a polyethelene catheter in the pericardial paracentesis. N Engl J Med 1955; 253: 872-3.

12.- Zahn E M, et al: Percutaneous pericardial catheter drainage in childhood. Am J Cardiol 1992; 70 (6): 678-80.

13.- Susini G: Percutaneous pericardiocentesis versus subxiphoid pericardotomy in cardiac tamponade due to postoperative pericardial effusion. Journal of Cardio-Thoracic Vascular Anesthesia 1993; 7 (2): 178-83.

14.- Henry J, Issenberg M: Pericardial effusion. En Pediatric Cardiac Intesive Care, W. Wilkins, Editor. 1998; pp. 510-1.

15.- Roodpeyma S, Sadeghian N: Acute pericarditis in childhood: A 10-year experience. Pediatr Cardiol 2000; 21: 363-7.

16.- Tsang TS, et al: Percutaneous echocardiographically guided pericardiocentesis in pediatric patients: evaluation of safety and efficacy. J Am Soc Echocardiogr 1998; 11 (11): 1072-7.

17.- Callahan JA, et al: Two-dimensional echocardiographically guided pericardiocentesis: experience in 117 consecutive patients. Am J Cardiol 1985; 55 (4): 476-9.

18.- Maggiolini $S$, et al: Echocardiography-guided pericardiocentesis with probe-mounted needle: report of 53 cases. J Am Soc Echocardiogr 2001; 14 (8): 821-4. 